\title{
State of sport in South Africa: Questions that need answers
}

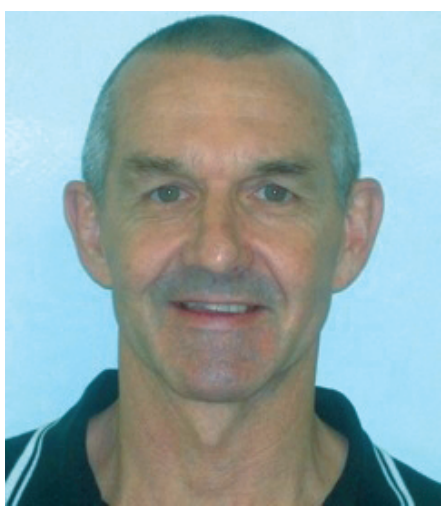

The application of science governing human performance in the laboratory is well refined with a defined set of guiding principles. For example, rules are in place about recruiting subjects, randomising them into different groups, familiarising them with the equipment used to measure performance, and then testing them before and after the implementation of the treatment. The implementation being evaluated may have something to do with nutrition, specialised training, tapering or pacing. Differences in the performance measurements conducted before and after the intervention are analysed using statistics, and conclusions can be made about whether or not there are differences as a result of the implementation of the treatment. The magnitude of the effect can be calculated and viable conclusions can be reached. Although the conclusions are open to interpretation, the findings are generally clear for all to see. This scenario describes the assessment of performance in the laboratory; how do we translate this set of principles and logic into evaluating the performances of our elite sportsmen and -women competing on the international platform? How can we determine the 'health' of a particular sporting code?

One possible way of quantifying performance is to consider medals won at the Olympics. After the London Olympics, much was made of South Africa's 23rd place and the 6 medals ( 3 gold, 2 silver and 1 bronze) of the South African athletes. This performance was much better than in Beijing (2008) (1 silver) and marginally better than Athens (2004) (1 gold, 3 silver and 2 bronze). However, it may be argued that the performance was on a par with the performance in Atlanta (1996) (3 gold, 1 silver and 1 bronze), achieved only two years into our new democracy. These criteria of evaluating overall performance are not absolute and the answer will vary depending on the agenda of the person doing the evaluation. Furthermore, when using only medals as a gauge of success, the athletes that achieved personal best performances, but only came 4 th in their event, are excluded from the count. Likewise those athletes that make the final, meaning they are in the approximate top 10 in the world, do not contribute to this simple medal count exercise. This type of assessment is also dependent on the state of the competitors from other countries - something that can clearly vary, as shown by Australia (10th place in 2012 with 35 medals dropping from 6th place and 46 medals in 2008). A much more robust way of assessing performance and the 'health' of a particular sporting code is to examine the sport at a more holistic level. Such a method will provide more meaningful results that can be monitored over time to determine any trends. The information can also be useful for assessing the efficacy of implementation, and whether money allocated to the sport is being spent in the most efficient way. Such a method is much more complicated than merely counting medals. For example, the following questions need answers before a firm conclusion about the state of the sport can be made.

- How many participants are there in the sport in the country?

- What are the demographics of the participants?

- What is the state of the facilities and equipment associated with that sport?

- What competitive leagues exist?

- Do these leagues have age-group competitions?

- Is there much attrition from the youth leagues to the adult leagues?

- How many coaches are fully engaged in the sport?

- Do these coaches have any formal training?

- How are talented athletes identified?

- Does the sporting code have a programme in place to nurture talent, and develop it fully through to adult levels?

- Are policies in place for those athletes who may get injured while participating in the sport?

- Is the Federation administered professionally and are succession plans in place for the administrators of the sport?

- How many participants are ranked in the top 10 in the world? In the top 50 ? In the top 100 ?

Answers to these questions are not easily obtained. However, monitoring systems need to be implemented so that an ongoing assessment of the sport can be made and the answers to these questions provided. Once that status is achieved will we be able to comment accurately on the status of sport in the country and whether we are improving or not.

This edition of the journal has two original research studies. The first paper examines the short-term effect of kinesio tape on the explosive power of the gluteus maximus of male athletes. This is an example of a good laboratory study, with findings that can be translated into practical applications. The second study examines the playing time of professional rugby players from 2007 to 2011 . The design of this study is aligned to the concept of monitoring the state of the game so that it can be managed more effectively. Lastly, the abstracts from the First International Conference of by the Biokinetics Association of South Africa to be held in Potchefstroom from 27 to 29 September 2012 are also presented in this edition. The broad scope of the content of the abstracts shows the important role that the profession of biokinetics is fulfilling. The authors of these studies are challenged to convert these abstracts into publications so that the information can be shared with a broader audience.

\section{Mike Lambert}

Editor-in Chief

S Afr J SM 2012;24(3):74. DOI:10.7196/SAJSM.345 